\title{
Two-photon Diffraction and Quantum Lithography
}

\author{
Milena D'Angelo, Maria V. Chekhova*, and Yanhua Shih \\ Department of Physics, University of Maryland, Baltimore County, Baltimore, Maryland 21250
}

\begin{abstract}
We report a proof-of-principle experimental demonstration of quantum lithography. Utilizing the entangled nature of a twophoton state, the experimental results have bettered the classical diffraction limit by a factor of two. This is a quantum mechanical two-photon phenomenon but not a violation of the uncertainty principle.
\end{abstract}

PACS Number: 42.50.Dv, 42.82.Cr, 42.25.Fx, 03.65.Bz

Classical optical lithography technology is facing its limit due to the diffraction effect of light. However, this classical limit can be surpassed, surprisingly, by utilizing the quantum nature of entangled multi-photon states [1]. In an idealized experimental situation, the minimum width of the entangled N-photon diffraction pattern can be $\mathrm{N}$ times narrower than the width of a classical diffraction pattern. The working principle of the effect has been discussed theoretically by Boto et al [2]; and by Scully from a different approach [3]. In particular, one can consider two-photon entangled states. For a twoparticle maximally entangled EPR state, the value of an observable for either single subsystem is indeterminate. However, if one subsystem is measured to be at a certain value for an observable, the value of that observable for the other subsystem is determined with certainty [1]. Because of this peculiar quantum nature, the two-photon diffraction pattern thus can be narrower than given by the classical limit under certain conditions. This effect has been experimentally observed by Kim and Shih [1.

We wish to report a proof-of-principle quantum lithography experiment in this letter. By using entangled photon pairs in Young's two-slit experiment, we found that under certain experimental conditions, the two-photon interference-diffraction pattern has spatial interference modulation period smaller and diffraction pattern width narrower, by a factory of two, than in the classical case.

One of the principles of the geometrical optics is that "light propagates in straight line". If this were always true, one could obtain the image of a physical object, for example, a physical slit, with an unlimitedly small size by applying a perfect lens system. Unfortunately, light is also a wave. The minimum size of the image one can make is determined by the wave property of the light: diffraction. The physics is very simple: according to Huygens's principle, each point on the primary wavefront serves as the source of spherical secondary amplitudes (wavelets) and advances with the speed and frequency equal to those of the primary wave. The wavelets from a physical slit will meet at any point in space with

\footnotetext{
*Permanent Address: Department of Physics, Moscow State University, Moscow, Russia.
}

different phases. The superposition of the wavelets will determine the size of the image. The intensity distribution of light can be calculated by considering an integral of the wavelets coming from the physical object.

Figure 11 schematically shows a classic one-dimensional optical diffraction by a single slit. A well collimated laser beam passes the slit and then its intensity distribution is analyzed in the Fourier transform plane (or in the farfield zone). This distribution, which is the diffraction pattern of a single slit, is well-known to be $\operatorname{sinc}^{2}(\beta)$, where the parameter $\beta=(\pi a / \lambda) \sin \theta \simeq(\pi a / \lambda) \theta$, $a$ is the width of the slit, and $\theta$ is the angle shown in Fig. 1 [5]. When $\beta$ reaches $\pi$, the superposition of the wavelets results in a minimum intensity. The $\operatorname{sinc}^{2}(\beta)$ pattern determines the minimum width one can obtain. Usually, this minimum width is called the "diffraction limit".

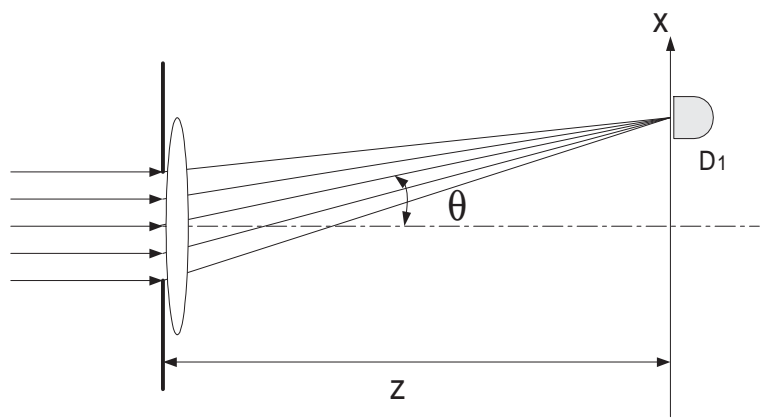

FIG. 1. Classical single-slit diffraction. Zero intensity occurs when the secondary waves ("amplitudes") interfere destructively.

To surpass the diffraction limit, our scheme is to utilize the entangled nature of an N-particle system. To understand the physics of this scheme, consider a gedankenexperiment which is illustrated in Fig. 2(a). An entangled photon pair can be generated anywhere in region $V$; however, photons belonging to the same pair can only propagate (1) oppositely and (2) "almost" horizontally (quantitative discussion will be given later) as indicated in the figure. Two slits are placed symmetrically on the left and right sides of the entangled photon source. A photon-counting detector is placed into the far-field zone (or the Fourier transform plane, if lenses are placed following the slits) on each side, and the coincidences between the "clicks" of both detectors are registered. The 
two detectors are scanning symmetrically, i.e., for each coincidence measurement, both detectors have equal $\mathrm{x}$ coordinates. A two-photon joint detection is the result of the superposition of the two-photon amplitudes, which are indicated in the figure by straight horizontal lines [6]. To calculate two-photon diffraction, we need to "superpose" all possible two-photon amplitudes. Different from the classical case, it is a double integral involving the two slits and the two-photon amplitudes (parallel lines in Fig. 2). The two-photon counterpart of the classical intensity, the joint detection counting rate, is now $\operatorname{sinc}^{2}(2 \beta)$, which gives narrower distribution than the classical pattern by a factor of two. Now if we "fold" the symmetrical left and right sides of the experimental setup together and replace the two independent detectors with a film that is sensitive only to two-photon light (two-photon transition material), then in principle, we have two-photon lithography.

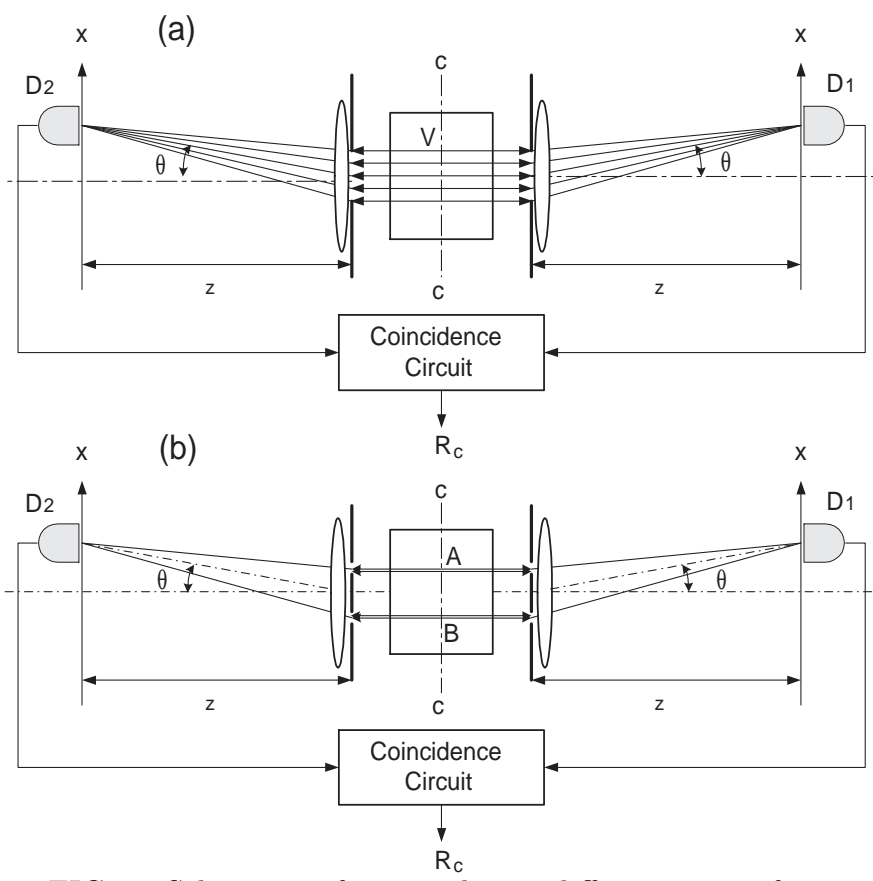

FIG. 2. Schematic of a two-photon diffraction-interference gedankenexperiment. The right and left sides of the picture represent signal and idler photons of an entangled pair. Detectors $D_{1}, D_{2}$ perform the joint detection (coincidence) measurement. It is easy to see that the superposition of the two-photon amplitudes results in twice faster in interference modulation and twice faster to approach the zero intensity of diffraction than in the case of Fig. 1.

If one replaces the single slit in the above setup with a double-slit, Fig. 2(b), it is also interesting to see that under the half-width diffraction pattern, the doubleslit two-photon spatial interference pattern has a higher modulation frequency, as if the wavelength of the light were reduced to one-half. To observe the two-photon interference, one has to "erase" the first-order interference by reinforcing an experimental condition: $\delta \theta>\lambda / b$ where $\delta \theta$ is the divergence of the light, $b$ is the distance between the two slits, and $\lambda$ is the wavelength.

The heart of this gedankenexperiment is a special twophoton source: the pair has to be generated in such a desired entangled way as described above. We have found and demonstrated that, under certain conditions, the two-photon state generated via spontaneous parametric down conversion (SPDC) satisfies the above requirements. The working principle, as well as the report of a real experiment, will be given below.

The schematic setup of the experiment is illustrated in Fig. 3. It is basically the "folded" version of a double-slit interference-diffraction experiment shown in Fig. 2(b).

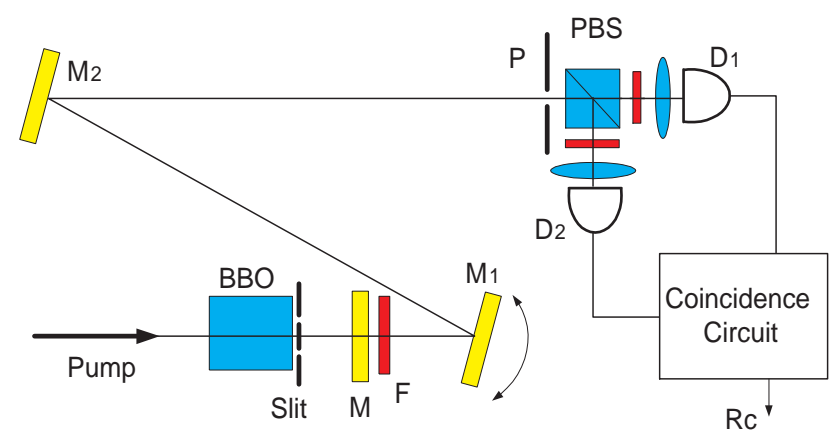

FIG. 3. Schematic of the experimental setup. Entangled photon pairs are produced in a BBO crystal and then pass through a double-slit placed immediately after the crystal. Detectors $D_{1}, D_{2}$ together with the pinhole P, the beamsplitter PBS, and the coincidence circuit represent a two-photon detector. The angle registered by this two-photon detector is scanned by rotating mirror $M_{1}$.

The $458 \mathrm{~nm}$ line of an Argon Ion laser is used to pump a $5 \mathrm{~mm}$ BBO $\left(\beta-\mathrm{BaB}_{2} \mathrm{O}_{4}\right)$ crystal, which is cut for degenerate collinear type-II phase matching [7 9] to produce pairs of orthogonally polarized signal (e-ray of the BBO) and idler (o-ray of the BBO) photons. Each pair emerges from the crystal collinearly, with $\omega_{s} \simeq \omega_{i} \simeq \omega_{p} / 2$, where $\omega_{j}(j=s, i, p)$ are the frequencies of the signal, idler, and pump, respectively. The pump is then separated from the signal-idler pair by a mirror $\mathrm{M}$, which is coated with reflectivity $R \simeq 1$ for the pump and transmissivity $T \simeq 1$ for the signal-idler. For further pump suppression, a cutoff filter $\mathrm{F}$ is used. The signal-idler beam passes through a double-slit [10], which is placed close to the output side of the crystal, and is reflected by two mirrors, $M_{1}$ and $M_{2}$, onto a pinhole $\mathrm{P}$ followed by a polarization beam splitter PBS. The signal and idler photons are separated by the beam splitter and are detected by the photon counting detectors $D_{1}$ and $D_{2}$, respectively. The output pulses of each detector are sent to a coincidence counting circuit with a $1.8 \mathrm{~ns}$ acceptance time window for the signal-idler joint detection. Both detectors are preceded by $10 \mathrm{~nm}$ bandwidth spectral filters centered at the degenerate wavelength, $916 \mathrm{~nm}$. The whole block containing the pinhole, PBS, the detectors, and the coincidence circuit can be considered as a two-photon detector. 
Instead of moving two detectors together as indicated in Fig. 2, we rotate the mirror $M_{1}$ to "scan" the spatial interference-diffraction pattern relative to the detectors.

One important point to be emphasized is that the double-slit must be placed as close as possible to the output surface of the BBO crystal. Only in this case, can the observed diffraction pattern be narrower than in the classical case by a factor of 2, see Eq. (9). Otherwise, it will be close to $\sqrt{ } 2$ as suggested in Ref. 3 .

Figure 1 reports the experimental results. In our experiment, the width of each slit is $a=0.13 \mathrm{~mm}$. The distance between the two slits is $b=0.4 \mathrm{~mm}$. The distance between the slits and the pinhole $\mathrm{P}$ is $4 \mathrm{~m}$. Figure shows the distribution of coincidences versus the rotation angle $\theta$ of mirror $M_{1}$. The spatial interference period and the first zero of the envelope are measured to be 0.001 and \pm 0.003 radians, respectively.

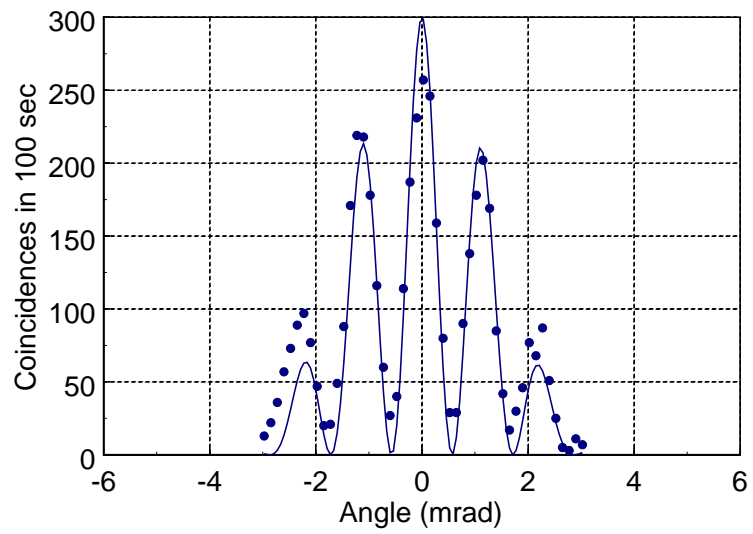

FIG. 4. Experimental measurement of a two-photon double-slit interference-diffraction pattern. Comparing with the classical case, it has a faster spatial interference modulation and a narrower diffraction pattern width, by a factor of 2 .

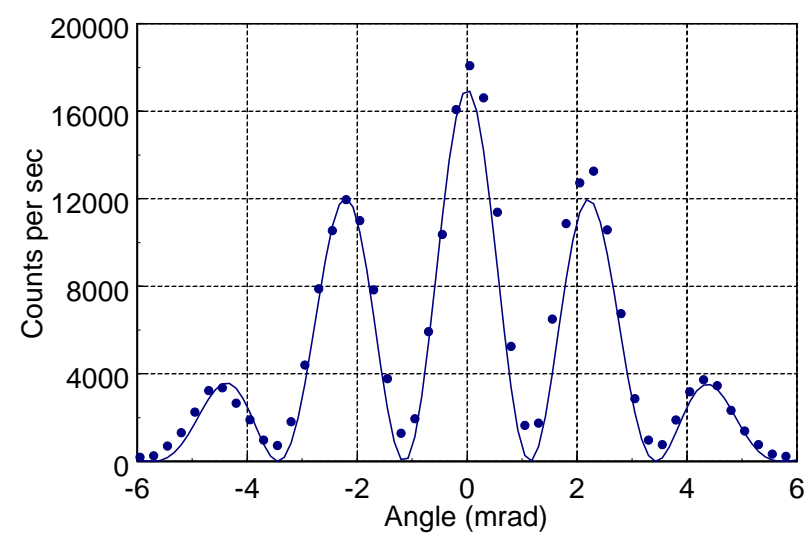

FIG. 5. Measurement of the interference-diffraction pattern for classical light in the same experimental setup.

For comparison, we also measured the first-order interference-diffraction pattern of a classical light with
$916 \mathrm{~nm}$ wavelength by the same double-slit in the same experimental setup, see Fig. 5. The spatial interference period and the first zero of the envelope are measured to be 0.002 and \pm 0.006 radians, respectively.

In both "classical" and "quantum" cases, we obtain similar standard Young's two-slit interference-diffraction pattern, $\operatorname{sinc}^{2}[(\pi a / \lambda) \theta] \cos ^{2}[(\pi b / \lambda) \theta]$; however, whereas the wavelength for fitting the curve in Fig. 5 (classical light) is $916 \mathrm{~nm}$, for the curve in Fig. 1 (entangled twophoton source) it has to be $458 \mathrm{~nm}$. Clearly, the twophoton diffraction "beats" the classical limit by a factor of 2 .

To be sure that we observed the effect of the SPDC photon pair with wavelength of $916 \mathrm{~nm}$ but not the pump laser beam with wavelength of $458 \mathrm{~nm}$, we remove or rotate the BBO crystal 90-degree to a non-phase-matching angle and examine the coincidence counting rate. The coincidences remain zero during the 100 second period, which is the data collection time duration for each of the data points, even in high power operation of the pump laser. Comparing this with the coincidence counting rate with BBO under phase-matching, see Fig. 4, there is no doubt that the observation is the effect due to the SPDC.

To explain the result, we have to take into account the quantum nature of the two-photon state. SPDC is a nonlinear optical process in which pairs of signal-idler photons are generated when a pump laser beam is incident onto an optical nonlinear material [7.8. Quantum mechanically, the state can be calculated by the first-order perturbation theory [7] and has the form

$$
|\Psi\rangle=\sum_{s, i} F\left(\omega_{s}, \omega_{i}, \mathbf{k}_{s}, \mathbf{k}_{i}\right) a_{s}^{\dagger}\left(\omega\left(\mathbf{k}_{s}\right)\right) a_{i}^{\dagger}\left(\omega\left(\mathbf{k}_{i}\right)\right)|0\rangle
$$

where $\omega_{j}, \mathbf{k}_{j}(\mathrm{j}=\mathrm{s}, \mathrm{i}, \mathrm{p})$ are the frequencies and wavevectors of the signal (s), idler (i), and pump (p), respectively, $F\left(\omega_{s}, \omega_{i}, \mathbf{k}_{s}, \mathbf{k}_{i}\right)$ is the so-called biphoton amplitude, the pump frequency and wavevector $\omega_{p}$ and $\mathbf{k}_{p}$ can be considered as constants, $a_{s}^{\dagger}$ and $a_{i}^{\dagger}$ are creation operators for the signal and idler photons, respectively. The biphoton amplitude contains $\delta$-functions of the frequency and wavevector,

$F\left(\omega_{s}, \omega_{i}, \mathbf{k}_{s}, \mathbf{k}_{i}\right) \propto \delta\left(\omega_{s}+\omega_{i}-\omega_{p}\right) \delta\left(\mathbf{k}_{s}+\mathbf{k}_{i}-\mathbf{k}_{p}\right)$.

The signal or idler photon could be in any modes of the superposition (uncertain); however, due to Eq. (2), if one photon is known to be in a certain mode then the other one is determined with certainty.

The phase matching conditions resulting from the $\delta$ functions in Eq. (2),

$$
\omega_{s}+\omega_{i}=\omega_{p}, \quad \mathbf{k}_{s}+\mathbf{k}_{i}=\mathbf{k}_{p},
$$

play important role in the experiment. The transverse component of the wavevector phase matching condition requires that

$$
k_{s} \sin \alpha_{s}=k_{i} \sin \alpha_{i},
$$


where $\alpha_{s}$ and $\alpha_{i}$ are the scattering angles inside the crystal. Upon exiting the crystal, Snell's law thus provides:

$$
\omega_{s} \sin \beta_{s}=\omega_{i} \sin \beta_{i}
$$

where $\beta_{s}$ and $\beta_{i}$ are the exit angles of the signal and idler with respect to $\mathbf{k}_{p}$ direction. Therefore, in the degenerate case, the signal and idler photons are emitted at equal, yet opposite, angles relative to the pump, and the measurement of the momentum (wavevector) of the signal photon determines the momentum (wavevector) of the idler photon with unit probability and vice versa. In the collinear case, which we use in our experiment, the scattering angles of the signal and idler photons are close to zero and occupy the range $\Delta \theta$, which is determined by the sizes of the crystal and the pump beam, see 11].

The coincidence counting rate $R_{c}$ is given by the probability $P_{12}$ of detecting the signal-idler pair by detectors $D_{1}$ and $D_{2}$ jointly,

$$
\begin{aligned}
P_{12} & =\left\langle\Psi\left|E_{1}^{(-)} E_{2}^{(-)} E_{2}^{(+)} E_{1}^{(+)}\right| \Psi\right\rangle \\
& =\left|\left\langle 0\left|E_{2}^{(+)} E_{1}^{(+)}\right| \Psi\right\rangle\right|^{2},
\end{aligned}
$$

where $|\Psi\rangle$ is the two-photon state of SPDC and $E_{1}, E_{2}$ are fields on the detectors. The effect of two-photon Young's interference can be easily understood if we assume for simplicity that signal and idler photons always go through the same slit and never go through different slits. This approximation holds if the variation of the scattering angle inside the crystal

$$
\Delta \theta \ll b / D,
$$

where $D$ is the distance between the input surface of the SPDC crystal and the double-slit. In this case, the state after the slits can be written as

$$
|\Psi\rangle=|0\rangle+\epsilon\left[a_{s}^{\dagger} a_{i}^{\dagger} \exp \left(i \varphi_{A}\right)+b_{s}^{\dagger} b_{i}^{\dagger} \exp \left(i \varphi_{B}\right)\right]|0\rangle,
$$

where $\epsilon \ll 1$ is proportional to the pump field and the nonlinearity of the crystal, $\varphi_{A}$ and $\varphi_{B}$ are the phases of the pump field at region A (upper slit) and region B (lower slit), and $a_{j}^{\dagger}\left(b_{j}^{\dagger}\right)$ are the photon creation operators for photons passing through the upper slit (A) and the lower slit (B). In our experiment, the ratio $(b / D) / \Delta \theta \simeq$ 30 and Eq. (7) is satisfied well enough. Moreover, even the ratio $(a / D) / \Delta \theta$ is of order of 10 , which satisfies the condition for observing two-photon diffraction:

$$
\Delta \theta \ll a / D .
$$

In Eq. (6), the fields on the detectors are given by:

$$
\begin{aligned}
& E_{1}^{(+)}=a_{s} \exp \left(i k r_{A 1}\right)+b_{s} \exp \left(i k r_{B 1}\right) \\
& E_{2}^{(+)}=a_{i} \exp \left(i k r_{A 2}\right)+b_{i} \exp \left(i k r_{B 2}\right)
\end{aligned}
$$

where $r_{A i}\left(r_{B i}\right)$ are the optical path lengths from region A (B) to the ith detector. Substituting Eqs. (8) and (10) into Eq. (6), we get

$$
\begin{aligned}
R_{c} \propto P_{12} & =\epsilon^{2}\left|\exp \left(i k r_{A}+i \varphi_{A}\right)+\exp \left(i k r_{B}+i \varphi_{B}\right)\right|^{2} \\
& \propto 1+\cos \left[k\left(r_{A}-r_{B}\right)\right],
\end{aligned}
$$

where we define $r_{A} \equiv r_{A 1}+r_{A 2}$. We have assumed $\varphi_{A}=$ $\varphi_{B}$ in Eq. (11).

In the far-field zone (or the Fourier transform plane), interference of the two amplitudes from Eq. (8) gives

$$
R_{c}(\theta) \propto \cos ^{2}[(2 \pi b / \lambda) \theta] .
$$

Eq. (12) has the form of a standard Young's two-slit interference pattern, except having the modulation period one-half of the classical case or an equivalent wavelength of $\lambda / 2$.

To calculate the diffraction effect of a single slit, we need an integral of the effective two-photon wavefunction over the slit width. Quite similarly to Eq. (12), it gives

$$
R_{c}(\theta) \propto \operatorname{sinc}^{2}[(2 \pi a / \lambda) \theta] .
$$

Eq. (13) has the form of standard single-slit diffraction pattern, except having half of the classical pattern width.

The combined interference-diffraction coincidence counting rate for the double-slit case is given by

$$
R_{c}(x) \propto \operatorname{sinc}^{2}[(2 \pi a / \lambda) \theta) \cos ^{2}[(2 \pi d / \lambda) \theta],
$$

which is a product of Eq. (12) and Eq. (13).

The experimental observations have confirmed the above quantum mechanical predictions.

In conclusion, we have demonstrated the possibility of quantum lithography by using an entangled two-photon state generated from a specially designed spontaneous parametric down conversion. One may not see advantages from the above proof-of-principle experimental demonstration. The results from the two-photon diffraction measurement is equivalent to that of using the pump laser beam directly. The advantage, however, is in the case of large number of entangled particle states. We have considered quantum lithography based on our entangled N-photon scheme $(N \geq 3)$ [12]. In these cases one can "beat" the classical limit by a factor of $N$ and keep the "pump" laser beam wavelength close to one-half that of the entangled photon beam.

The authors thank Y.H. Kim, J.P. Dowling, D.V. Strekalov, M.O. Scully and H.S. Pilloff for helpful discussions. This work was supported, in part, by ARDANSA, ONR, and NSF. M.V. Chekhova acknowledges the support from the Russian Foundation for Basic Research, grant No. 99-02-16419.

[1] A. Einstein, B. Podolsky, and N. Rosen, Phys. Rev. 47, 777 (1935).

[2] A.N. Boto et al, Phys. Rev. Lett., 85, 2733 (2000). 
[3] M.O. Scully, Presented at Conference on "Effects of Atomic Coherence and Interference in Quantum Optics", Crested Butte, Colorado, (1993); In his presentation, Scully proposed a two-photon microscope to narrow down the classical limit a factor of $\sqrt{ } 2$.

[4] Y.-H. Kim and Y.H. Shih, Foundations of Physics, Vol. 29, 1849 (1999).

[5] See classical optics textbooks, for example, E. Hecht, $O p$ tics, Addison-Wesley Publishing, 2nd Edition (1989).

[6] T.B. Pittman et al, Phys. Rev. A 52, R3429 (1995); D.V. Strekalov et al, Phys. Rev. Lett., 74, 3600 (1995).

[7] D.N. Klyshko, Photons and Nonlinear Optics, Gordon and Breach Science, New York, (1988). In pioneer works, spontaneous parametric down conversion was called "Spontaneous Parametric Fluorescence" and "Spontaneous Parametric Scattering".

[8] A. Yariv, Quantum Electronics, John Wiley and Sons, New York, (1989).

[9] In type-I SPDC, signal and idler are both ordinary (or extraordinary) rays of the crystal; however, in type-II SPDC they are orthogonally polarized, i.e., one is ordinary and the other is extraordinary.

[10] We use a double-slit instead of a single slit since the interference-diffraction pattern has more details in this case and is therefore more "recognizable".

[11] A.V.Burlakov, M.V.Chekhova, D.N.Klyshko, A.N.Penin, Y.H.Shih, and D.V.Strekalov, Phys.Rev.A, Vol. 56, 3214 (1997).

[12] T.E. Keller, M.H. Rubin, Y.H. Shih, and L.A. Wu, Phys. Rev. A, Vol. 57, 2076 (1998). 